\title{
Generalized Fibonacci Polynomials and some Identities
}

\author{
G. P. S. Rathore \\ Department of Mathematics, \\ College of Horticulture, \\ Mandsaur, (M. P.) India
}

\author{
Omprakash Sikhwal \\ Devanshi Tutorial, Keshaw \\ Kunj, Mandsaur (M.P.), India
}

\author{
Ritu Choudhary \\ School of Studies in \\ Mathematics, Vikram \\ University Ujjain (M.P.), India
}

\begin{abstract}
The Fibonacci polynomials and Lucas polynomials are famous for possessing wonderful and amazing properties and identities. Generalization of Fibonacci polynomial has been done using various approaches. One usually found in the literature that the generalization is done by varying the initial conditions. In this paper, Generalized Fibonacci polynomials are defined by $w_{n}(x)=x w_{n-1}(x)+w_{n-2}(x) ; n \geq 2$ with $w_{0}(x)=2 b$ and $w_{1}(x)=a+b$, where $\mathrm{a}$ and $\mathrm{b}$ are integers. Further, some basic identities are generated and derived by generating function.
\end{abstract}

\section{Keywords}

Fibonacci polynomial, Lucas polynomial, Generalized Fibonacci polynomial, Generating function.

\section{Mathematics Subject Classification 2010 11B37, 11B39}

\section{INTRODUCTION}

The Fibonacci polynomials and Lucas polynomials are famous for possessing wonderful and amazing properties and identities. Fibonacci polynomials appear different frameworks .These polynomials are of great importance in the study of many subjects such as algebra, geometry, combinatorics, approximation theory, statistics and number theory itself. Moreover these polynomials have been applied in every branch of mathematics. Fibonacci polynomials are special cases of chebyshev polynomials and have been studies on more advanced level by many mathematicians.

Basin [1] show that Q matrix generates a set of Fibonacci Polynomials satisfying the recurrence relation

$f_{n}(x)=x f_{n-1}(x)+f_{n-2}(x), n \geq 2$ with $f_{0}(x)=0, f_{l}(x)=1$

The first few polynomials of (1.1) are

$f_{1}(x)=1, f_{2}(x)=x, f_{3}(x)=x^{2}+1, f_{4}(x)=x^{3}+2 x$,

$f_{5}(x)=x^{4}+3 x^{2}+1, \quad f_{6}(x)=x^{5}+4 x^{3}+3 x$ and so on.

The Lucas polynomials [10] are defined by

$l_{n}(x)=x l_{n-1}(x)+l_{n-2}(x), n \geq 2$ with $l_{0}(x)=2, l_{1}(x)$

Generating function of Fibonacci polynomials is

$\sum_{n=0}^{\infty} f_{n}(x) t^{n}=t\left(1-x t-t^{2}\right)^{-1}$

Generating function of Lucas polynomials is

$\sum_{n=0}^{\infty} l_{n}(x) t^{n}=(2-x t)\left(1-x t-t^{2}\right)^{-1}$.
Explicit sum formula for (1.1) is given by

$$
f_{n}(x)=\sum_{k=0}^{\left[\frac{n-1}{2}\right]}\left(\begin{array}{c}
n-k-1 \\
k
\end{array}\right) x^{n-1-2 k} .
$$

Where $\left(\begin{array}{l}n \\ k\end{array}\right)$ is binomial coefficient and $[\mathrm{X}]$ is the greatest integer less than or equal to $\mathrm{X}$.

Explicit sum formula for (1.2) is given by

$l_{n}(x)=\sum_{k=0}^{\left[\frac{n}{2}\right]} \frac{n}{n-k}\left(\begin{array}{c}n-k \\ k\end{array}\right) x^{n-2 k}$.

Where $\left(\begin{array}{l}n \\ k\end{array}\right)$ is binomial coefficient and $[\mathrm{X}]$ is the greatest integer less than or equal to $\mathrm{X}$.

The Fibonacci and Lucas polynomials are many fascinating properties which have been studied in [2] to [12].

In this paper, we present generalized Fibonacci polynomials by varying the initial conditions. Further, some basic identities and derived by generating function.

\section{GENERALIZED FIBONACCI POLYNOMIALS}

Generalized Fibonacci polynomials have been intensively studied for many years and have become an interesting topic in Applied Mathematics. Generalization of Fibonacci polynomial has been done using various approaches. One usually found in the literature that the generalization is done by varying the initial conditions.

Generalized Fibonacci polynomials $w_{n}(x)$ are defined by recurrence relation

$$
\begin{aligned}
& w_{n}(x)=x w_{n-1}(x)+w_{n-2}(x) ; n \geq 2 . \text { with } w_{0}(x)=2 b, \\
& w_{1}(x)=a+b, \text { where a and } \mathrm{b} \text { are integers. }
\end{aligned}
$$

The first few terms of generalized Fibonacci polynomials are as follows:

$$
\begin{aligned}
& w_{0}(x)=2 b, \\
& w_{1}(x)=a+b, \\
& w_{3}(x)=x(a+b)+2 b, \\
& w_{4}(x)=(a+b) x^{2}+2 b x+(a+b) \text { and so on. }
\end{aligned}
$$

If $\mathrm{x}=1$, then $w_{n}(1)$ is Generalized Fibonacci sequence.

Generating function of Generalized Fibonacci polynomials is 
$\sum_{n=0}^{\infty} w_{n}(x) t^{n}=\frac{2 b(1-x t)+(a+b) t}{\left(1-x t-t^{2}\right)}$

\section{Hypergeometric representation}

$\sum_{n=0}^{\infty} w_{n}(x) t^{n}=[2 b(1-x t)+(a+b) t]\left(1-x t-t^{2}\right)^{-1}$

$=[2 b(1-x t)+(a+b) t]\left[(1-(x+t) t]^{-1}\right.$

$=[2 b(1-x t)+(a+b) t] \sum_{n=0}^{\infty}(x+t)^{n} t^{n}$

$=[2 b(1-x t)+(a+b) t] \sum_{n=0}^{\infty} t^{n} \sum_{k=0}^{n}\left(\begin{array}{l}n \\ k\end{array}\right) x^{n-k} t^{k}$

$=[2 b(1-x t)+(a+b) t] \sum_{n=0}^{\infty} \sum_{k=0}^{n} \frac{\lfloor\underline{n}}{\underline{k}\lfloor n-k} x^{n-k} t^{n+k}$

$=[2 b(1-x t)+(a+b) t] \sum_{n=0}^{\infty} \sum_{k=0}^{\infty} \frac{\underline{\underline{h}+k}}{\underline{\underline{k}\lfloor n}} x^{n} t^{n+2 k}$

$=[2 b(1-x t)+(a+b) t] \sum_{n=0}^{\infty} \frac{(x t)^{n}}{\lfloor n} \sum_{k=0}^{\infty} \frac{\lfloor n+k}{\underline{k}} t^{2 k}$

$=[2 b(1-x t)+(a+b) t] e^{x t} \sum_{k=0}^{\infty} \frac{\underline{h+k}}{\underline{\underline{n}}} \frac{\left(t^{2}\right)^{k}}{\underline{k}}$

$=[2 b(1-x t)+(a+b) t] e^{x t} \sum_{k=0}^{\infty} \frac{\sqrt{n+k+1}}{\sqrt{n+1}} \frac{\left(t^{2}\right)^{k}}{\lfloor k}$

$=[2 b(1-x t)+(a+b) t] e^{x t} \sum_{k=0}^{\infty} \frac{(n+1)_{k}(1)_{m}}{(1)_{m}} \frac{\left(t^{2}\right)^{k}}{\lfloor k}$

$=[2 b(1-x t)+(a+b) t] e^{x t}{ }_{2} F_{1}\left(n+1,1 ; 1 ; t^{2}\right)$

Hence hypergeometric representation of generating function is

$\sum_{n=0}^{\infty} \frac{w_{n}(x) t^{n}}{\lfloor n}$

$=[2 b(1-x t)+(a+b) t] e^{x t}{ }_{2} F_{1}\left(n+1,1 ; 1 ; t^{2}\right)$.

\section{SOME IDENTITIES OF}

\section{GENERALIZED FIBONACCI} POLYNOMIAL

In this section, some basic identities of Generalized Fibonacci polynomials have been obtained by method of generating function.

\section{Theorem 3.1. Prove that}

$w_{n+1}(x)-w_{n-1}(x)=x w_{n}(x), n \geq 1$.

Proof. By generating function of Generalized Fibonacci polynomial,

$\sum_{n=0}^{\infty} w_{n}(x) t^{n}=[2 b(1-x t)+(a+b) t]\left(1-x t-t^{2}\right)^{-1}$.

Differentiating both side with respect to $t$, $\sum_{n=0}^{\infty} n w_{n}(x) t^{n-1}=[2 b(1-x t)+(a+b) t](x+2 t)\left(1-x t-t^{2}\right)^{-2}+(a+b-2 b x)\left(1-x t-t^{2}\right)^{-1}$

$\left(1-x t-t^{2}\right) \sum_{n=0}^{\infty} n w_{n}(x) t^{n-1}$

$=[2 b(1-x t)+(a+b) t](x+2 t)\left(1-x t-t^{2}\right)^{-1}+(a+b-2 b x)$

$=(x+2 t) \sum_{n=0}^{\infty} w_{n}(x) t^{n}+(a+b-2 b x)$.

$\sum_{n=0}^{\infty} n w_{n}(x) t^{n-1}-\sum_{n=0}^{\infty} x n w_{n}(x) t^{n}-\sum_{n=0}^{\infty} n w_{n}(x) t^{n+1}$

$=\sum_{n=0}^{\infty} x w_{n}(x) t^{n}+2 \sum_{n=0}^{\infty} w_{n}(x) t^{n+1}+(a+b-2 b x)$.

Now, equating the coefficient of $\mathrm{t}^{\mathrm{n}}$ on both side,

$(n+1) w_{n+1}(x)-n x w_{n}(x)-(n-1) w_{n-1}(x)=x w_{n}(x)+2 w_{n-1}(x)$,

$(n+1) w_{n+1}(x)-(n+1) w_{n-1}(x)=(n+1) x w_{n}(x)$,

$w_{n+1}(x)-w_{n-1}(x)=x w_{n}(x)$.

Theorem 3.2. Prove that

$w_{n}^{\prime}(x)=x w_{n-1}^{\prime}(x)+w_{n-2}^{\prime}(x)+w_{n-1}(x), n \geq 2$.

Proof. By generating function of Generalized Fibonacci polynomial,

$\sum_{n=0}^{\infty} w_{n}(x) t^{n}=[2 b(1-x t)+(a+b) t]\left(1-x t-t^{2}\right)^{-1}$.

Differentiating both sides with respect to $\mathrm{x}$,

$\sum_{n=0}^{\infty} w_{n}^{\prime}(x) t^{n}=[2 b(1-x t)+(a+b) t] t\left(1-x t-t^{2}\right)^{-2}+(-2 b t)\left(1-x t-t^{2}\right)^{-1}$,

$\left(1-x t-t^{2}\right) \sum_{n=0}^{\infty} w_{n}^{\prime}(x) t^{n}=[2 b(1-x t)+(a+b) t] t\left(1-x t-t^{2}\right)^{-1}+(-2 b t)$,

$\left(1-x t-t^{2}\right) \sum_{n=0}^{\infty} w_{n}^{\prime}(x) t^{n}=t \sum_{n=0}^{\infty} w_{n}(x) t^{n}-2 b t$,

$\sum_{n=0}^{\infty} w_{n}^{\prime}(x) t^{n}-\sum_{n=0}^{\infty} x w_{n}^{\prime}(x) t^{n+1}-\sum_{n=0}^{\infty} w_{n}^{\prime}(x) t^{n+2}=\sum_{n=0}^{\infty} w_{n}^{\prime}(x) t^{n+1}-2 b t$.

Now, equating the coefficient of $\mathrm{t}^{\mathrm{n}}$ on both sides.

$w_{n}^{\prime}(x)=x w_{n-1}^{\prime}(x)+w_{n-2}^{\prime}(x)+w_{n-1}(x)$.

Theorem 3.3. Prove that

$w_{n+1}^{\prime}(x)=x w_{n}^{\prime}(x)+w_{n}(x)+w_{n-1}^{\prime}(x), n \geq 1$.

Proof. By (3.1),

$w_{n+1}(x)-w_{n-1}(x)=x w_{n}(x), n \geq 1$.

By differentiating with respect to $\mathrm{x}$,

$w_{n+1}^{\prime}(x)-w_{n-1}^{\prime}(x)=x w_{n}^{\prime}(x)+w_{n}(x)$,

$w_{n+1}^{\prime}(x)=x w_{n}^{\prime}(x)+w_{n}(x)+w_{n-1}^{\prime}(x)$.

Theorem 3.4. Prove that

$n w_{n}(x)=x w_{n}^{\prime}(x)+2 w_{n-1}^{\prime}(x), n \geq 1$ and

$x w_{n+1}^{\prime}(x)=(n+1) w_{n+1}(x)-2 w_{n}^{\prime}(x), n \geq 1$.

Proof. By generating function of Generalized Fibonacci polynomials, 
$\sum_{n=0}^{\infty} w_{n}(x) t^{n}=[2 b(1-x t)+(a+b) t]\left(1-x t-t^{2}\right)^{-1}$.

Differentiating both sides with respect to t,

$\sum_{n=0}^{\infty} n w_{n}(x) t^{n-1}$

$=[2 b(1-x t)+(a+b) t](x+2 t)\left(1-x t-t^{2}\right)^{-2}+(a+b-2 b x)\left(1-x t-t^{2}\right)^{-1}$.

Differentiating both sides with respect to $\mathrm{x}$,

$\sum_{n=0}^{\infty} w_{n}^{\prime}(x) t^{n}$

$=[2 b(1-x t)+(a+b) t] t\left(1-x t-t^{2}\right)^{-2}+(-2 b t)\left(1-x t-t^{2}\right)^{-1}$.

$\sum_{n=0}^{\infty} w_{n}^{\prime}(x) t^{n-1}$

$=[2 b(1-x t)+(a+b) t]\left(1-x t-t^{2}\right)^{-2}-2 b\left(1-x t-t^{2}\right)^{-1}$.

$\sum_{n=0}^{\infty} w_{n}^{\prime}(x) t^{n-1}+2 b\left(1-x t-t^{2}\right)^{-1}$

$=[2 b(1-x t)+(a+b) t]\left(1-x t-t^{2}\right)^{-2}$.

Using (3.5) in (3.4),

$\sum_{n=0}^{\infty} n w_{n}(x) t^{n-1}$

$=(x+2 t)\left\{\sum_{n=0}^{\infty} w_{n}^{\prime}(x) t^{n-1}+2 b\left(1-x t-t^{2}\right)^{-1}\right\}+(a+b-2 b x)\left(1-x t-t^{2}\right)^{-1}$

$=(x+2 t) \sum_{n=0}^{\infty} w_{n}^{\prime}(x) t^{n-1}+(x+2 t) 2 b\left(1-x t-t^{2}\right)^{-1}+(a+b-2 b x)\left(1-x t-t^{2}\right)^{-1}$.

$\sum_{n=0}^{\infty} n w_{n}(x) t^{n-1}$

$=x \sum_{n=0}^{\infty} w_{n}^{\prime}(x) t^{n-1}+2 \sum_{n=0}^{\infty} w_{n}^{\prime}(x) t^{n}+(x+2 t) 2 b\left(n 1-x t-t^{2}\right)^{-1}$

$+(a+b-2 b x)\left(1-x t-t^{2}\right)^{-1}$.

Equating the coefficient of $\mathrm{t}^{\mathrm{n}-1}$ on both sides,

$n w_{n}(x)=x w_{n}^{\prime}(x)+2 w_{n-1}^{\prime}(x)$.

(3.6)

Again equating the coefficient of $\mathrm{t}^{\mathrm{n}}$ on both sides,

$x w_{n+1}^{\prime}(x)=(n+1) w_{n+1}(x)-2 w_{n}^{\prime}(x)$.

\section{Theorem 3.5. Prove that}

$(n+1) w_{n}(x)=w_{n+1}^{\prime}(x)+w_{n-1}^{\prime}(x), n \geq 1$.

Proof. By (3.1),

$w_{n+1}(x)-w_{n-1}(x)=x w_{n}(x)$.

By differentiating with respect to $\mathrm{x}$,

$w_{n+1}^{\prime}(x)-w_{n-1}^{\prime}(x)=x w_{n}^{\prime}(x)+w_{n}(x)$.

Using equation (3.6) in equation (3.8),

$(n+1) w_{n}(x)=w_{n+1}^{\prime}(x)+w_{n-1}^{\prime}(x)$.

\section{Theorem 3.6. Prove that}

$x w_{n}^{\prime}(x)=2 w_{n+1}^{\prime}(x)-(n+2) w_{n}(x), n \geq 0$.
$2 w_{n+1}^{\prime}\left((n+1) w_{n}(x)=w_{n+1}^{\prime}(x)+\frac{1}{2}\left[n w_{n}(x)-x w_{n}^{\prime}(x)\right]\right.$.

$\left.2(n+1) w_{n}(x)=x\right)+n w_{n}(x)-x w_{n}^{\prime}(x)$,

$x w_{n}^{\prime}(x)=2 w_{n+1}^{\prime}(x)-(n+2) w_{n}(x)$.

\section{Theorem 3.7. Prove that}

$(n+1) x w_{n}^{\prime}=n w_{n+1}^{\prime}(x)-(n+2) w_{n-1}^{\prime}(x), n \geq 1$.

Proof. Using equation (3.3) in equation (3.9),

$$
\begin{aligned}
& (n+1)\left\{w_{n+1}^{\prime}(x)-x w_{n}^{\prime}(x)-w_{n-1}^{\prime}(x)\right\}=w_{n+1}^{\prime}(x)+w_{n-1}^{\prime}(x), \\
& n w_{n+1}^{\prime}(x)-(n+2) w_{n-1}^{\prime}(x) \\
& =(n+1) x w_{n}^{\prime}(x) .
\end{aligned}
$$

Theorem 3.8. (Explicit sum formula): For Generalized Fibonacci Polynomials,

$w_{n}(x)=2 b \sum_{k=0}^{\left[\frac{n}{2}\right]}\left(\begin{array}{c}n-k \\ k\end{array}\right) x^{n-2 k}$.

Proof. By generating function,

$$
\begin{aligned}
& \sum_{n=0}^{\infty} w_{n}(x) t^{n}=[2 b(1-x t)+(a+b) t]\left(1-x t-t^{2}\right)^{-1}, \\
& =[2 b(1-x t)+(a+b) t] \sum_{n=0}^{\infty}(x+t)^{n} t^{n}, \\
& =[2 b(1-x t)+(a+b) t] \sum_{n=0}^{\infty} t^{n} \sum_{k=0}^{n}\left(\begin{array}{l}
n \\
k
\end{array}\right) x^{n-k} t^{k}, \\
& =[2 b(1-x t)+(a+b) t] \sum_{n=0}^{\infty} \sum_{k=0}^{n} \frac{\lfloor n}{\underline{k} \underline{n} n-k} x^{n-k} t^{n+k}, \\
& =[2 b(1-x t)+(a+b) t] \sum_{n=0}^{\infty} \sum_{k=0}^{\infty} \frac{\underline{n} \underline{n} \underline{k} \underline{n}}{\underline{k}} x^{n} t^{n+2 k} .
\end{aligned}
$$

Equating coefficient of $\mathrm{t}^{\mathrm{n}}$ on both sides,

$$
w_{n}(x)=2 b \sum_{k=0}^{\left[\frac{n}{2}\right]}\left(\begin{array}{c}
n-k \\
k
\end{array}\right) x^{n-2 k} \text {. }
$$

Theorem 3.9. For positive integer $n \geq 0$, prove that

$$
w_{n}(x)=2 b x_{2}^{n} F_{1}\left(\left(\frac{-n}{2}, \frac{-n+1}{2},-n, \frac{-4}{x^{2}}\right)\right. \text {. }
$$

Proof. By (3.12), 


$$
\begin{aligned}
w_{n}(x) & =2 b x^{n} \sum_{k=0}^{\left[\frac{n}{2}\right]} \frac{\lfloor n-k}{\lfloor k n-2 k} x^{-2 k} \\
& =2 b x^{n} \sum_{k=0}^{\left[\frac{n}{2}\right]} \frac{(-1)^{k}(1)_{n}(-n)_{2 k}}{(-n)_{k}(-1)^{2 k}(1)_{n}} \frac{x^{-2 k}}{\lfloor k} \\
& =2 b x^{n} \sum_{k=0}^{\left[\frac{n}{2}\right]} \frac{(-1)^{k} 2^{2 k}\left(\frac{-n}{2}\right)_{k}\left(\frac{-n+1}{2}\right)_{k} \frac{x^{-2 k}}{\underline{k}}}{(-n)_{k}(-1)^{2 k}} \\
& =2 b x^{n} \sum_{k=0}^{\left[\frac{n}{2}\right]} \frac{(-1)^{k}\left(\frac{-n}{2}\right)_{k}\left(\frac{-n+1}{2}\right)_{k}\left(\frac{-4}{x^{2}}\right)^{k}}{(-n)_{k} \mid k} . \\
w_{n}(x) & =2 b x^{n}{ }_{2} F_{1}\left(\left(\frac{-n}{2}, \frac{-n+1}{2},-n, \frac{-4}{x^{2}}\right) .\right.
\end{aligned}
$$

Theorem 3.10. For positive integer $n \geq 0$, prove that

$$
\begin{aligned}
& \sum_{n=0}^{\infty}(c)_{n} w_{n}(x) \frac{t^{n}}{\underline{n}} \\
& =2 b(1-x t)^{-c}{ }_{3} F_{2}\left(\frac{c}{2}, \frac{c+1}{2}, n+1, \frac{n+1}{2}, \frac{n+2}{2}, \frac{t^{2}}{(1-x t)^{2}}\right) .
\end{aligned}
$$

Proof. Multiplying both sides of (3.12) by $(c)_{n} \frac{t^{n}}{\lfloor n}$ and summing between the limit $n=0$ and $n=\infty$,

$$
\begin{aligned}
& \sum_{n=0}^{\infty}(c)_{n} w_{n}(x) \frac{t^{n}}{\lfloor n} \\
& =2 b \sum_{n=0}^{\infty} \sum_{k=0}^{\left[\frac{n}{2}\right\rfloor} \frac{\lfloor n-k}{\lfloor\underline{k n-2 k}}(c)_{n} x^{n-2 k} \frac{t^{n}}{\lfloor n}, \\
& =2 b \sum_{n=0}^{\infty} \sum_{k=0}^{\infty} \frac{\underline{n+k}}{\underline{k} \underline{n} \underline{n+2 k}}(c)_{n+2 k} x^{n} t^{n+2 k}, \\
& =2 b \sum_{n=0}^{\infty} \sum_{k=0}^{\infty} \frac{\underline{n+k}}{\underline{k} \underline{n} \underline{n+2 k}}(c+2 k)_{n}(c)_{2 k}(x t)^{n} t^{2 k} \text {, } \\
& =2 b\left\{\sum_{n=0}^{\infty}(c+2 k)_{n} \frac{(x t)^{n}}{\lfloor n}\right\} \sum_{k=0}^{\infty} \frac{\lfloor n+k}{\lfloor\underline{n} n+2 k}(c)_{2 k} t^{2 k} \text {, } \\
& =2 b(1-x t)^{-(c+2 k)} \sum_{k=0}^{\infty} \frac{\lfloor n+k}{\lfloor\underline{n+2 k}}(c)_{2 k} t^{2 k}, \\
& =2 b(1-x t)^{-c} \sum_{k=0}^{\infty} \frac{\frac{\underline{\underline{n+k}}}{\underline{\underline{\underline{n}}}}}{\frac{\underline{n+2 k}}{\lfloor n}}(2)^{2 k}\left(\frac{c}{2}\right)_{k}\left(\frac{c+1}{2}\right)_{k}\left[\frac{t^{2}}{(1-x t)^{2}}\right]^{k} \text {, }
\end{aligned}
$$

$$
\begin{aligned}
& =2 b(1-x t)^{-c} \sum_{k=0}^{\infty} \frac{(n+1)_{k}}{(n+1)_{2 k}}(2)^{2 k}\left(\frac{c}{2}\right)_{k}\left(\frac{c+1}{2}\right)_{k}\left[\frac{t^{2}}{(1-x t)^{2}}\right]^{k}, \\
& =2 b(1-x t)^{-c} \sum_{k=0}^{\infty} \frac{(n+1)_{k}}{(2)^{2 k}\left(\frac{n+1}{2}\right)_{k}\left(\frac{n+2}{2}\right)_{k}}(2)^{2 k}\left(\frac{c}{2}\right)_{k}\left(\frac{c+1}{2}\right)_{k}\left[\frac{t^{2}}{(1-x t)^{2}}\right]^{k}, \\
& =2 b(1-x t)^{-c} \sum_{k=0}^{\infty} \frac{\left(\frac{c}{2}\right)_{k}\left(\frac{c+1}{2}\right)_{k}(n+1)_{k}}{\left(\frac{n+1}{2}\right)_{k}\left(\frac{n+2}{2}\right)_{k}}\left[\frac{t^{2}}{(1-x t)^{2}}\right]^{k}, \\
& \sum_{n=0}^{\infty}(c)_{n} w_{n}(x) \frac{t^{n}}{\lfloor n} \\
& =2 b(1-x t)^{-c}{ }_{3} F_{2}\left(\frac{c}{2}, \frac{c+1}{2}, n+1, \frac{n+1}{2}, \frac{n+2}{2}, \frac{t^{2}}{(1-x t)^{2}}\right) .
\end{aligned}
$$

\section{CONCLUSION}

In this paper, Generalized Fibonacci polynomials are introduced by varying the initial conditions. Further, some basic identities established and derived by standard methods.

\section{ACKNOWLEDGMENTS}

The authors are thankful to the reviewers for their constructive suggestions and comments for improving the exposition of the original version.

\section{REFERENCES}

[1] Basin, S. L., The appearance of Fibonacci numbers and the Q Matrix in Electrical Network Theory Magazine, Vol. 36, No. 2, (1963) 84-90.

[2] Bicknell, Marjorie. A Primer for the Fibonacci Number: part and $7^{\text {th }}-$ An introduction to Fibonacci polynomials their Divisibility properties, The Fibonacci Quarterly, Vol. 8, No.4 (1970), 407-420.

[3] Doman, B. G. S. and Williams ,J. K.., Fibonacci and Lucas Polynomials, Mathematical Proceedings of the Cambridge Philosophical Society, 90,Part 3 (1981), 385387.

[4] Glasson, Alan R., Remainder Formulas, Involving Generalized Fibonacci and Lucas Polynomials, The Fibonacci Quarterly, vol. 33, No. 3,(1995),36-39.

[5] Hayes, Richard A., Fibonacci and Lucas polynomials, Master's Thesis, San Jose State college, January, (1965), 36-39.

[6] Hoggatt, V. E. Jr., Private communication of Nov. 17, 1965 to Selmo Tauber, The Fibonacci Quarterly, Vol. 6, (1968), 99.

[7] Hoggatt, V. E. Jr. and Long, C. T., Divisibility properties of Fibonacci Polynomials, The Fibonacci Quarterly, Vol. 12, No. 2, (1974),113-120.

[8] Horadam, A. F., Mahon, J. M., Pell-Lucas Polynomials, The Fibonacci Quarterly, Vol. 23,No. 1 (1985),7-20.

[9] Koshy, T., Fibonacci and Lucas Number with application, John Wiley and Sons. New York, 2001.

[10] Lupas A., A Guide of Fibonacci and Lucas Polynomials, Octagon Mathematics Magazine, Vol. 7, No. 1 (1999), 212. 
[11] Singh B., Bhatnagar S., Sikhwal O., Fibonacci-Like Polynomials and Identities, International Journal of Advanced Mathematical Sciences, 1 (3) (2013), 152-157.

[12] Singh M., Sikhwal O., and Gupta Y., Generalized Fibonacci-Lucas Polynomials, International Journal of Advanced Mathematical Sciences ,2(1)(2014), 81-87.
[13] Singh B., Sikhwal O., Bhatnagar S., Fibonacci-Like Sequence and its Properties, Int. J. Contemp. Math. Sciences, Vol. 5,No. 18, (2010), 859-868.

[14] Singh B., Sikhwal O., and Panwar Y. K., Determinantal Identities Involving Lucas Polynomials, Applied Mathematical Sciences Vol. 3, (2009), No. 8, 377-388. 